\title{
The effect of primary fixation with standard postfixation and the duration of hydrolysis on nuclear features in image cytometry
}

\author{
Margareta Fležar $^{\mathrm{a}, *}$, Alexei Doudkine ${ }^{\mathrm{b}}$ and Marija Us-Krašovec ${ }^{\mathrm{a}}$ \\ ${ }^{a}$ Department Cytopathology, Institute of Oncology, 1000 Ljubljana, Slovenia \\ ${ }^{\mathrm{b}}$ Oncometrics Imaging Corp., Vancouver, BC, Canada V5Z 1L3
}

Received 16 December 1997

Revised 2 October 1998

Accepted 15 October 1998

\begin{abstract}
The effect of three primary fixation procedures, used in the preparation of routine cytological samples: air-drying, Delaunay, and Saccomanno fixation, with postfixation in modified Böhm-Sprenger fixative, on nuclear features as a function of hydrolysis time is reported. Three different cell types: lymphatic cells (tonsil), epithelial cells (buccal mucosa) and mesenchymal cells (uterine myometrium) were used for the study. Our findings show, that generally not all features have the same plateau times as the IOD (integrated optical density), and that many features show different values depending on cell type and fixation method. It is therefore recommended that for any primary fixative used in routine clinical work and for each cell type, the hydrolysis curve for all nuclear features to be used in sample analysis should be established.
\end{abstract}

Keywords: Image cytometry, fixation, Feulgen reaction, hydrolysis, nuclear texture features

\section{Introduction}

Image cytometry is becoming a commonly used tool in clinical, mostly retrospective, studies. Initially, the emphasis has been placed on the measurements of the DNA amount in the cells, which is commonly analysed by absorbance image cytometry of cells stained with DNA specific, stoichiometric stains.

Over the last decade, with the introduction of high resolution CCD (Charge-Coupled Device) cameras and powerful computers, besides DNA amount also other nuclear features, characterizing the DNA distribution in cell nuclei became the focus of interest. These properties are best expressed with texture features [6] which can be roughly divided into descriptive statistics of chromatin distribution, discrete texture features, Markovian texture features, fractal texture features and run length texture features. Texture features have been proposed to provide additional (and possibly superior) information which may be of both diagnostic and prognostic significance.

The values of any nuclear feature strongly depend on fixation procedures, hydrolysis procedures, cell type, instrumentation, etc. Great emphasis has been placed on these issues in measurements of DNA amount (integrated optical density - IOD) of cell nuclei but not in nuclear texture features. For Feulgen

\footnotetext{
*Corresponding author: Margareta Fležar, MD, MSc, Department Cytopathology, Institute of Oncology, Zaloška 2, 1105 Ljubljana, Slovenia. Tel.: +1 3866113220 99; Fax: +1 386611314 180; E-mail: mflezar@onko-i.si.
} 
based DNA staining, which is considered by many as the mandatory for absorbance-based DNA measurements, acid hydrolysis is a required step to open sites for DNA staining and a hydrolysis curve is recommended to be established for any tissue (cell) type and fixative. It has been established that different cell types exhibit different hydrolysis curves which are obtained by measurements of the IOD as a function of time of hydrolysis in the acid bath at a constant temperature [17,20]. In most cases, the IOD hydrolysis curve reaches a plateau after $45-60 \mathrm{~min}$ at room temperature using $5 \mathrm{M} \mathrm{HCl}$ and remains relatively constant for 30-60 min afterward. Thereafter, the IOD values will decrease with further hydrolysis due to the initiation of the breakdown of the DNA.

Relatively little attention has been paid to the effect of hydrolysis on texture features. In the literature we found only a single report dealing with this issue [9]. However, even in this work the authors investigated single fixatives (no postfixation) employed immediately before the Feulgen reaction.

In our work reported here, we examined the effect of three different fixatives, commonly used in the routine preparation of cytological samples: air-drying, Delaunay fixative, and Saccomanno fixative with postfixation in modified Böhm-Sprenger fixative, on 5 representative nuclear features. Their effect was studied in three different cell types: lymphatic cells, mesenchymal cells, and epithelial cells. We were particularly concerned to measure the nuclear feature values in the plateau range of the IOD hydrolysis curve, as this range will, in most cases, be used in clinical investigations or routine clinical practice.

\section{Materials and methods}

For this study we prepared cell samples of fresh tissues from tonsil, uterus, and buccal mucosa. The effects of fixation on lymphatic cells were studied on 72 imprints from cut surfaces of one tonsil that was therapeutically removed by tonsillectomy. The effects of fixation on epithelial cells were studied on 72 smears of buccal mucosa obtained from healthy volunteers. The effects of fixation on mesenchymal cells were studied on 72 smears prepared from myometrium of a surgically removed uterus for nonmalignant disease. Fixation protocol was identical for all tissue types. Twenty-four cell samples of each tissue type were air-dried, 24 samples were immediately fixed in Delaunay fixative and 24 were fixed in Saccomanno fixative (Table 1). The samples were left in the selected fixation solution overnight, in the morning they were removed from the fixative, dried and stored at $4{ }^{\circ} \mathrm{C}$ in the dark until staining.

Table 1

Chemical composition of fixatives and duration of fixation in different fixation techniques

\begin{tabular}{lll}
\hline Fixation & Chemical composition of fixative & Duration of fixation \\
\hline $\begin{array}{l}\text { Primary fixation: } \\
\text { Air drying }\end{array}$ & $18-22 \mathrm{~h}$ \\
Saccomanno & $98 \mathrm{vol} \%$ ethanol (50\%) $2 \mathrm{vol} \%$ polyethylene-glycol (1500 Merck) & $18-22 \mathrm{~h}$ \\
Delaunay & $500 \mathrm{ml}$ ethanol (96\%) & $18-22 \mathrm{~h}$ \\
& $500 \mathrm{ml}$ acetone \\
& $0.5 \mathrm{ml} 1 \mathrm{M} \mathrm{TCA}$ & \\
Postfixation: & & $30-140 \mathrm{~min}$ \\
Modified Böhm-Sprenger & $640 \mathrm{ml}$ methanol & \\
& $120 \mathrm{ml}$ formaldehyde (37\% stock solution) & \\
\hline
\end{tabular}


All samples were postfixed in modified Böhm-Sprenger fixative (Table 1) for 30 to $140 \mathrm{~min}$ immediately before the hydrolysis, as a part of the modified Feulgen staining procedure. Hydrolysis was performed in $5 \mathrm{M} \mathrm{HCl}$ at room temperature for $10,20,30,45,60,75,90$ and $120 \mathrm{~min}$, respectively.

After hydrolysis, the samples were stained by a modified Feulgen method using thionin staining (thionin acetate, colour index 52000, Lot No. MY 00209 TW, Aldrich Chemical Company, Inc., Milwaukee, USA) for $60 \mathrm{~min}$ [22].

Image analysis of cell samples was performed employing an automated, high resolution image cytometer (Cyto-Savant, Oncometrics Imaging Corp., Vancouver, BC, Canada). The system is equipped with high resolution CCD camera (Xillix Technologies Corp., Richmond, BC, Canada), bright field microscope, $x-y-z$ motorized stage, automated slide loader, computer and controllers. The images of the nuclei are projected onto a $1320 \times 1035$ pixel scientific CCD positioned in the primary image plane of a $20 \times$ Plan-Apochromatic objective lens (N.A. 0.75). A $600 \pm 10 \mathrm{~nm}$ filter is used because it corresponds to the spectral region of peak absorption of the nuclear stain. The individual sensing elements of the CCD are $6.8 \mu \mathrm{m} \times 6.8 \mu \mathrm{m}$ squares and sense light on their entire surface (100\% fill factor). At the $20 \times$ magnification, each sensing element covers approximately $0.1 \mu \mathrm{m}^{2}(0.34 \mu \mathrm{m} \times 0.34 \mu \mathrm{m})$ square area of the nucleus. Standard shading correction and correction for the local background are incorporated in the system. The hardware and its performance are described in detail elsewhere $[8,10]$.

Five hundred (500) nuclei per cell sample were acquired automatically by random sampling by means of the data acquisition program Acquire, incorporated into the system. All nuclear images were acquired in the best focus and the edge relocation algorithm was applied for precise segmentation of nuclei from the background [14]. From the gallery of the acquired images, we manually excluded all artifacts, images of degenerated nuclei, images of double or multiple overlapping nuclei or nuclei that were not in precise focus.

Over 100 nuclear features were calculated from each of the nuclear images. Nuclear features included common morphometric features (area, diameter, shape features, etc.), descriptive statistics of chromatin distribution (integrated optical density (IOD), variance of optical density (OD), OD skewness and kurtosis), discrete chromatin distribution (area and shape of high, medium, low density chromatin components, average distance between chromatin components of the same optical densities, etc.), Markovian texture features (entropy, energy, cluster shade, etc.), local extreme features (number of local minima and maxima in the image) and run length texture features (short run emphasis, long run emphasis, gray level nonuniformity, etc.). Exact formulas and more complete feature descriptions can be found in the references [6]. Only nuclear features of the cells from $\mathrm{G}_{0} / \mathrm{G}_{1}$ phase of the cell cycle were included in the statistical analysis. Data were imported into the BMDP statistical software package for further analysis. Hydrolysis curves were obtained for all tissue types and for all fixation conditions. The plateau times for IOD were found to be between 60 and $90 \mathrm{~min}$ of hydrolysis for all tissue types and fixatives (Fig. 1). Statistical significance of any differences in nuclear features of the cells with the same fixation, but with different hydrolysis times in the plateau range were tested with ANOVA (Tukey studentized rang test). Also, the differences of the cells with different fixation but the same hydrolysis time were tested. The size of each cell group was between 1000 and 1500 objects (nuclei). The differences were considered statistically significant at $p<0.05$.

\section{Results}

The analysis of variance showed significant differences in the values of nuclear features between the differently treated cells of the same tissue type. 

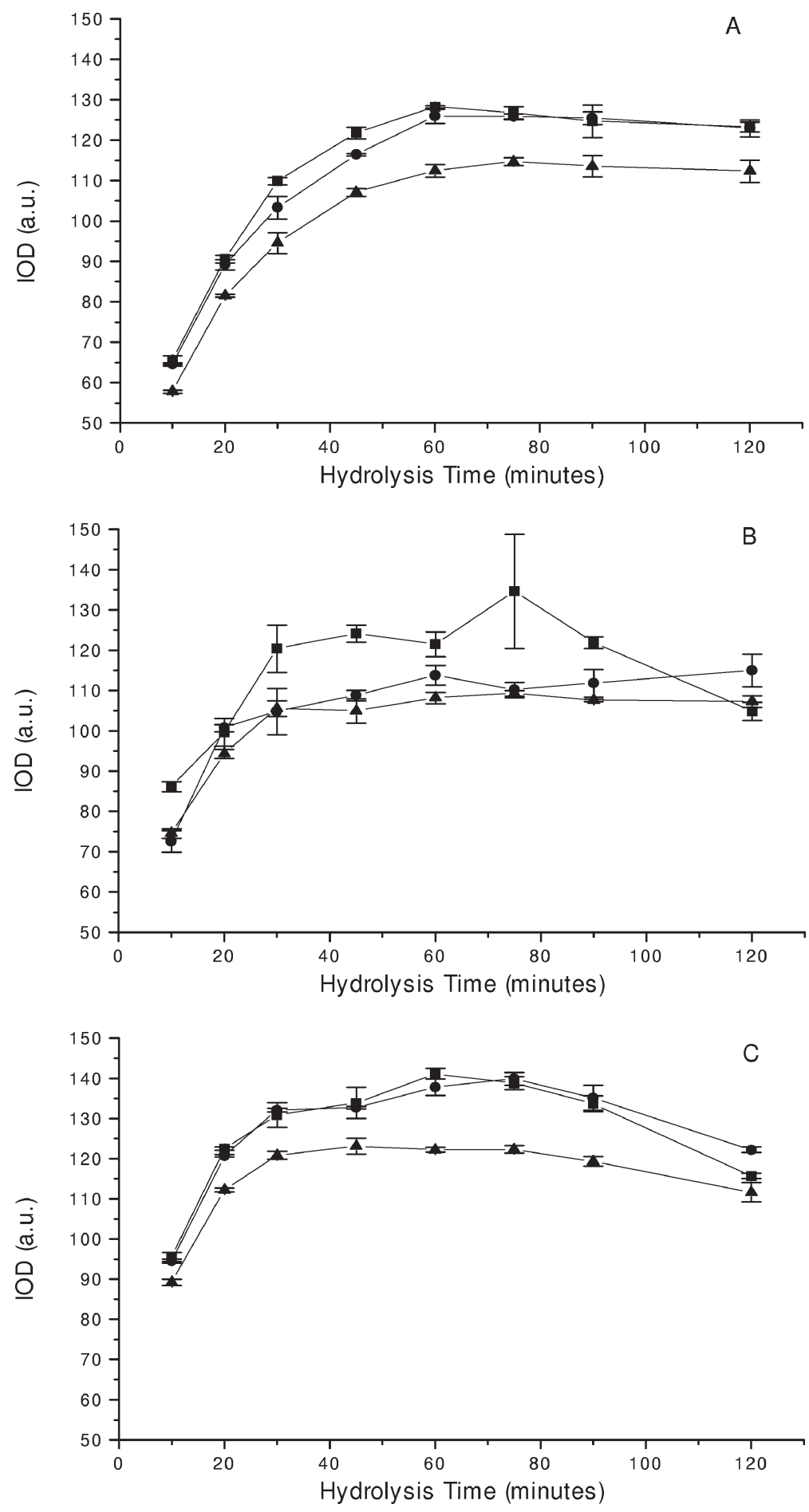

Fig. 1. Hydrolysis curves for lymphatic cells (A), epithelial cells from buccal mucosa (B), uterine myometrial cells (C) fixed by different types of fixatives: - Delaunay, $\bullet-$ Saccomanno, $\boldsymbol{\Delta}$ - air-drying with postfixation in modified Böhm-Sprenger fixative. 


\subsection{Morphometric features: nuclear area}

We found that the nuclear area of lymphatic cells (Fig. 2A) was constant between 60 and 75 min of hydrolysis. The area of epithelial cells (Fig. 2B) was constant over all of the plateau times, but the area of mesenchymal cells (Fig. 2C) changed between different hydrolysis times of the plateau range.

The alcohol fixed (Delaunay and Saccomanno) lymphatic cells had significantly smaller nuclear area than air-dried (Fig. 2A). In contrast, the nuclear area of alcohol fixed mesenchymal cells was larger than area of air-dried mesenchymal cells (Fig. 2C). Each of the three fixation procedures gave diverse nuclear areas of epithelial cells (Fig. 2B).

\subsection{Discrete texture features: high density objects}

This feature measures the number of high density clumps in the nucleus. The values of high density objects of epithelial cells (Fig. 3B), mesenchymal cells (Fig. 3C) for all fixations and Delaunay fixed lymphatic cells (Fig. 3A) were constant over the IOD plateau times. The values of this feature were variable in Saccomanno fixed and air-dried lymphatic cells (Fig. 3A).

Significant differences were found between alcohol fixed (Saccomanno and Delaunay) and air-dried nuclei of lymphatic (Fig. 3A) and mesenchymal cells (Fig. 3C). Alcohol fixed lymphatic cells (Fig. 3A) and air-dried mesenchymal cells (Fig. 3C) had highest values of this feature. Air-dried and Saccomanno fixed epithelial cells had similar values, which were significantly higher than values of Delaunay fixed epithelial cells (Fig. 3B).

\subsection{Markovian texture features: entropy}

Entropy measures a "disorder" of chromatin distribution, yielding high values for spatially disorganized chromatin structure without distinct clumps. Entropy values were constant for all tissue types and fixations (Fig. 4), except for air-dried mesenchymal cells (Fig. 4C) during 60 and $75 \mathrm{~min}$ of the IOD plateau.

We found significant differences between all three fixations in most of the hydrolysis times, although the differences were smaller or not even significant between Saccomanno and Delaunay fixed nuclei (Fig. 4). The air-dried nuclei had the highest values of entropy in all three tissue types.

\subsection{Fractal dimension}

Fractal dimension yields high values to nuclear images with spatially frequent, high contrast variations in chromatin condensation. The values of fractal dimension were constant between 60 and 75 min of hydrolysis for air-dried and for Delaunay fixed lymphatic cells (Fig. 5A), for epithelial cells (all fixations) (Fig. 5B) and for Saccomanno fixed and for air-dried mesenchymal cells (Fig. 5C). Longer hydrolysis gave variable values of this feature.

We found significant differences between the three fixations at the same hydrolysis time in lymphatic and in mesenchymal cells, with the exception that there were no differences between Delaunay fixed and air-dried lymphatic nuclei at 60 and $90 \mathrm{~min}$, and no difference between the alcohol fixed mesenchymal cells at $75 \mathrm{~min}$. Epithelial cells had similar values for all three fixations. 

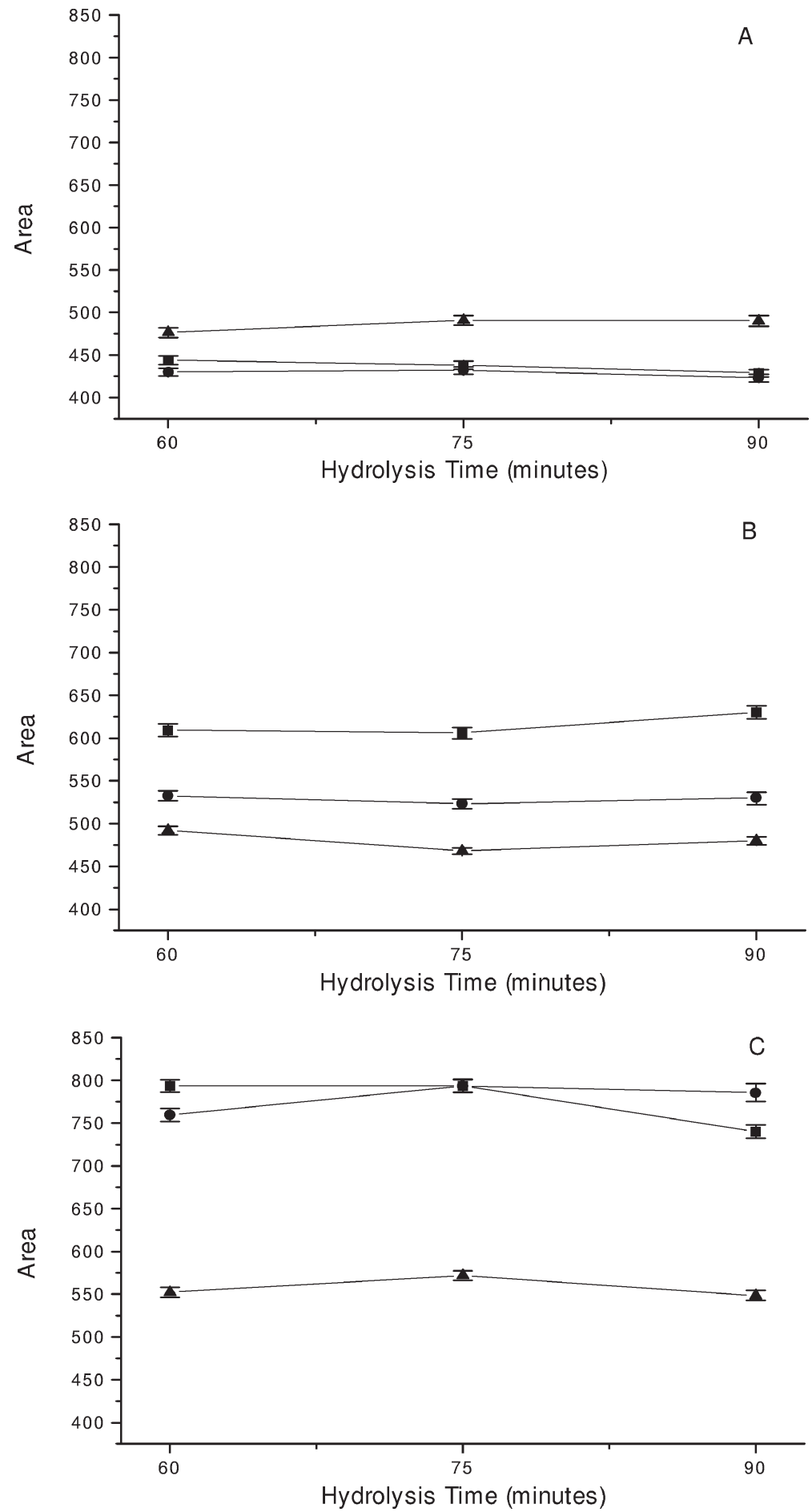

Fig. 2. Mean values $( \pm \mathrm{SEM})$ of nuclear area in the plateau range of hydrolysis curve for lymphatic cells (A), epithelial cells from buccal mucosa (B) and uterine myometrial cells (C) fixed by different types of fixatives: $\mathbf{a}$ - Delaunay, $\bullet$ - Saccomanno, $\Delta$ - air-drying with postfixation in modified Böhm-Sprenger fixative. 

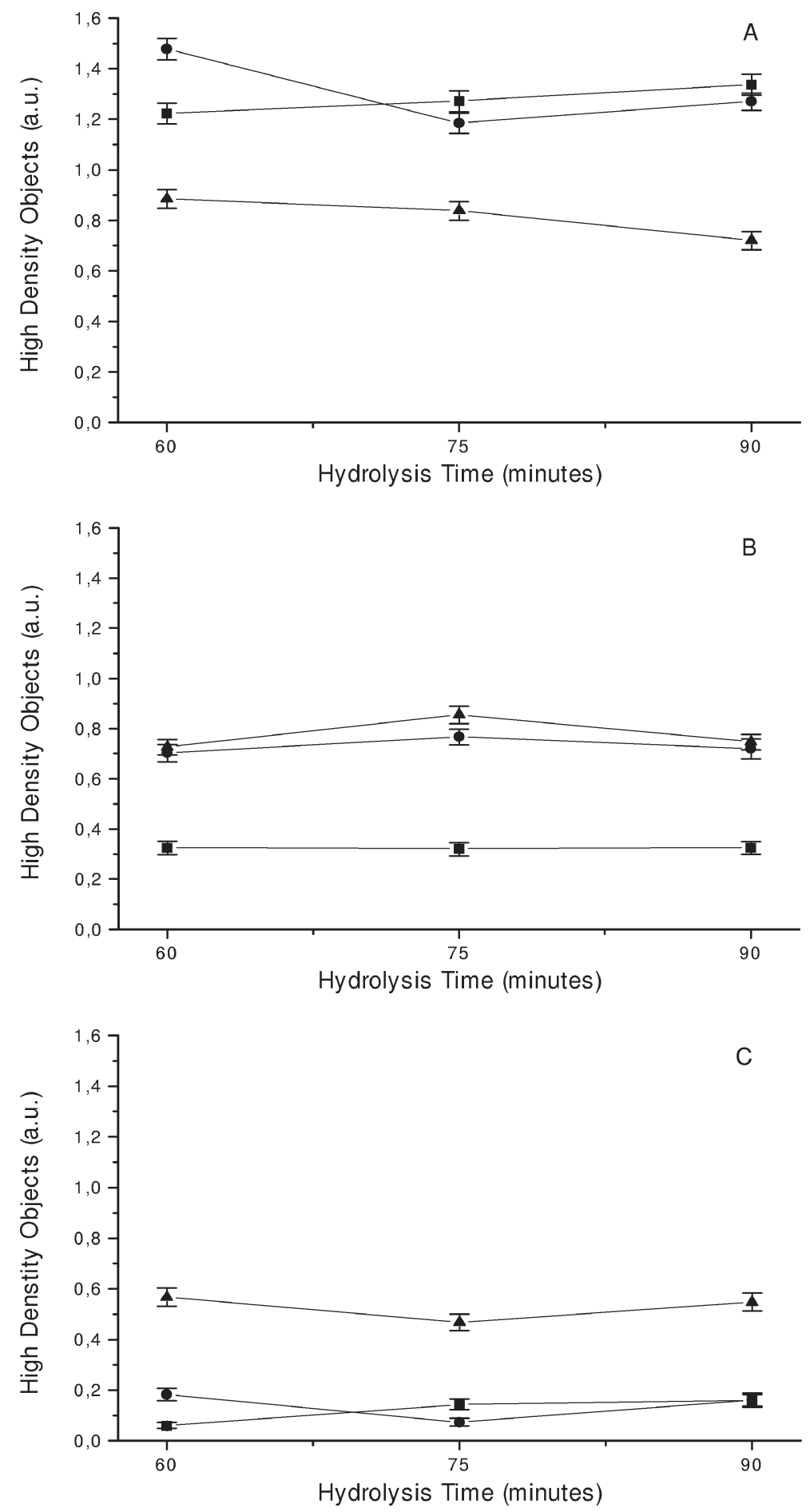

Fig. 3. Mean values $( \pm$ SEM) of high density objects in the plateau range of hydrolysis curve for lymphatic cells (A), epithelial cells from buccal mucosa (B) and uterine myometrial cells (C) fixed by different types of fixatives:

- - Saccomanno, $\boldsymbol{\Delta}$ - air-drying. 

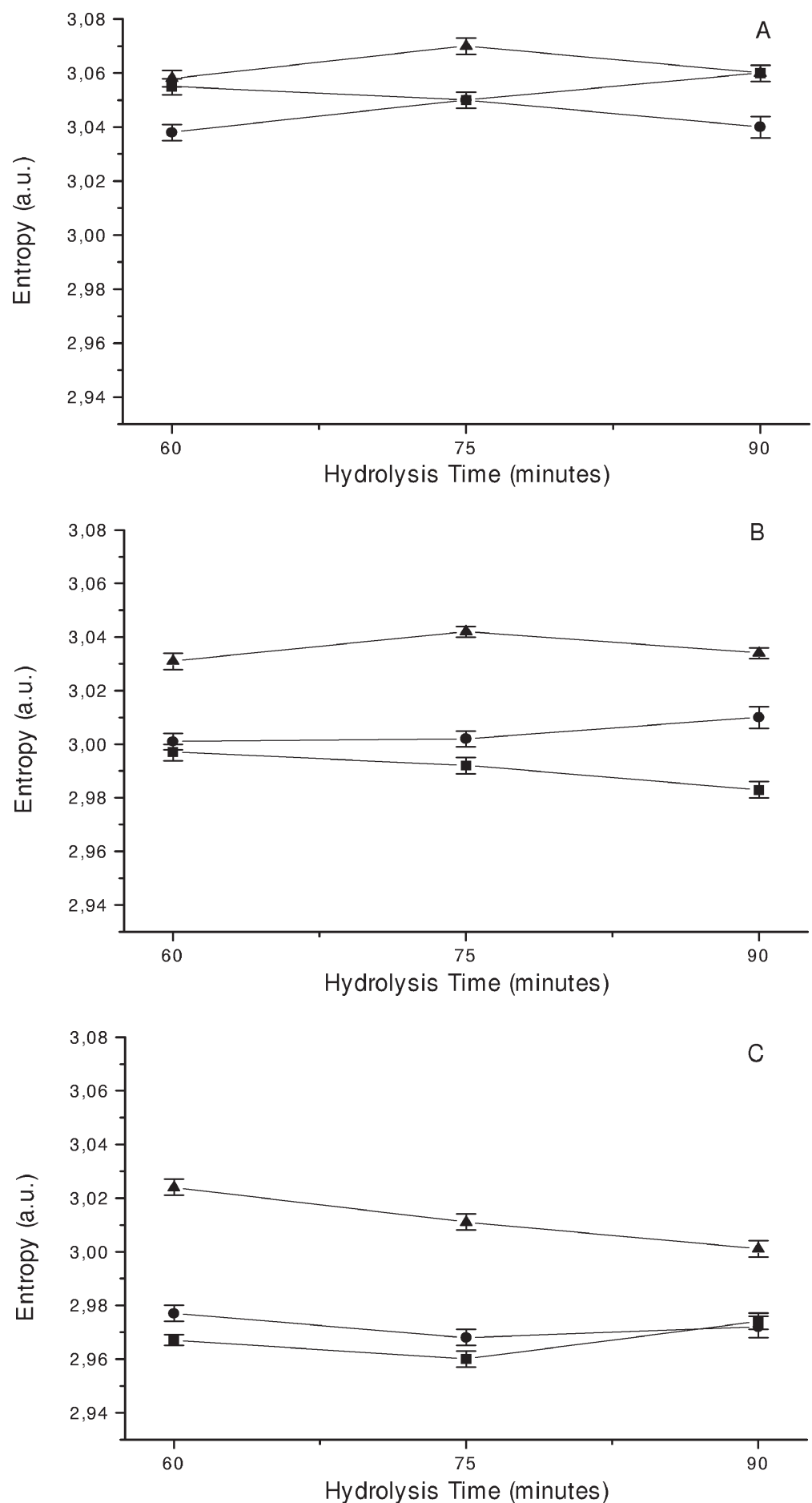

Fig. 4. Mean values ( \pm SEM) of entropy in the plateau range of hydrolysis curve for lymphatic cells (A), epithelial cells from buccal mucosa (B) and uterine myometrial cells (C) fixed by different types of fixatives: $\Delta$ - air-drying with postfixation in modified Böhm-Sprenger fixative. 

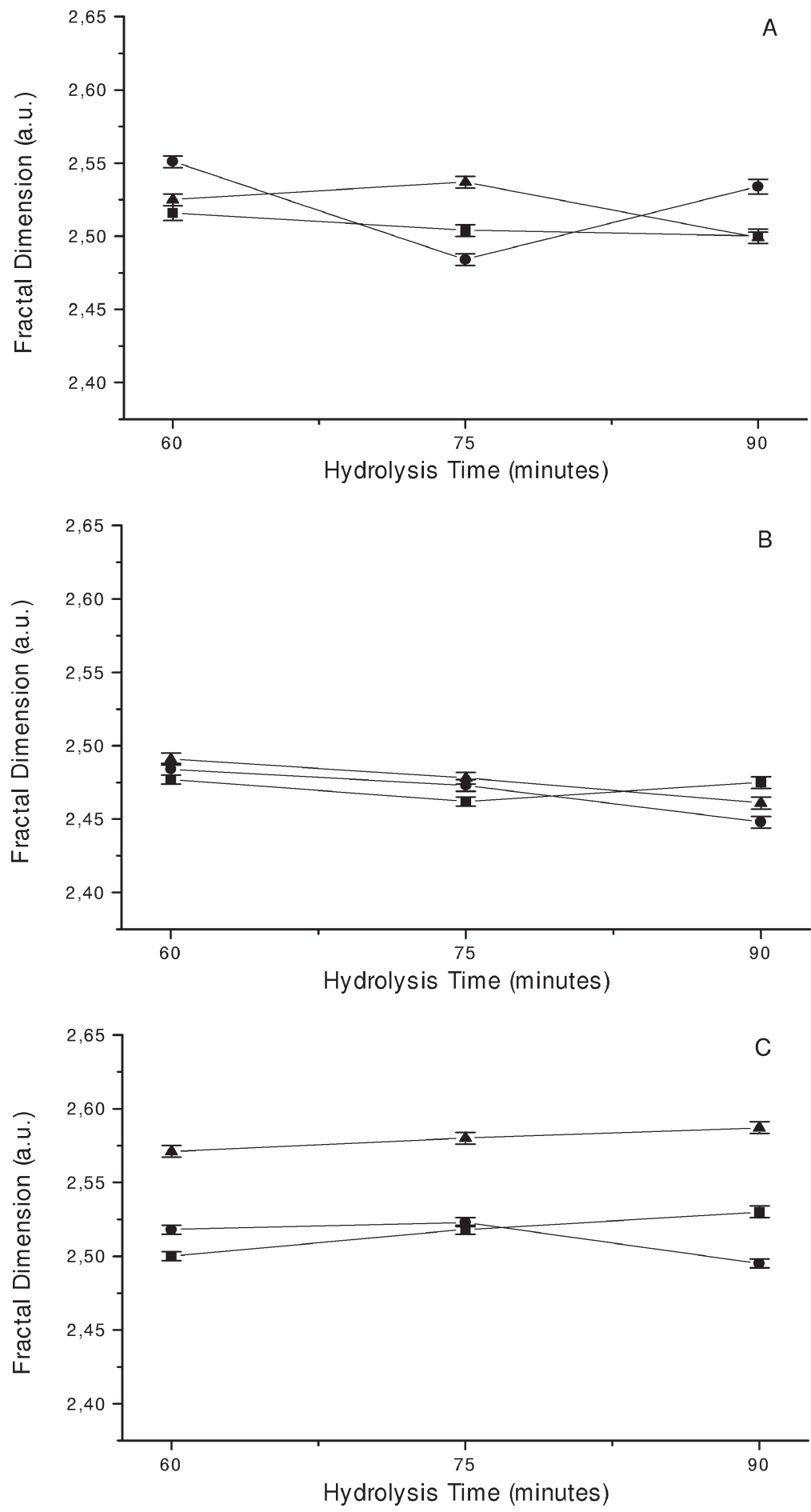

Fig. 5. Mean values $( \pm$ SEM) of fractal dimension in the plateau range of hydrolysis curve for lymphatic cells (A), epithelial cells from buccal mucosa (B) and uterine myometrial cells (C) fixed by different types of fixatives: $\mathbf{\square}$ - Delaunay, $\bullet-$ Saccomanno, $\mathbf{\Lambda}$ - air-drying with postfixation in modified Böhm-Sprenger fixative. 

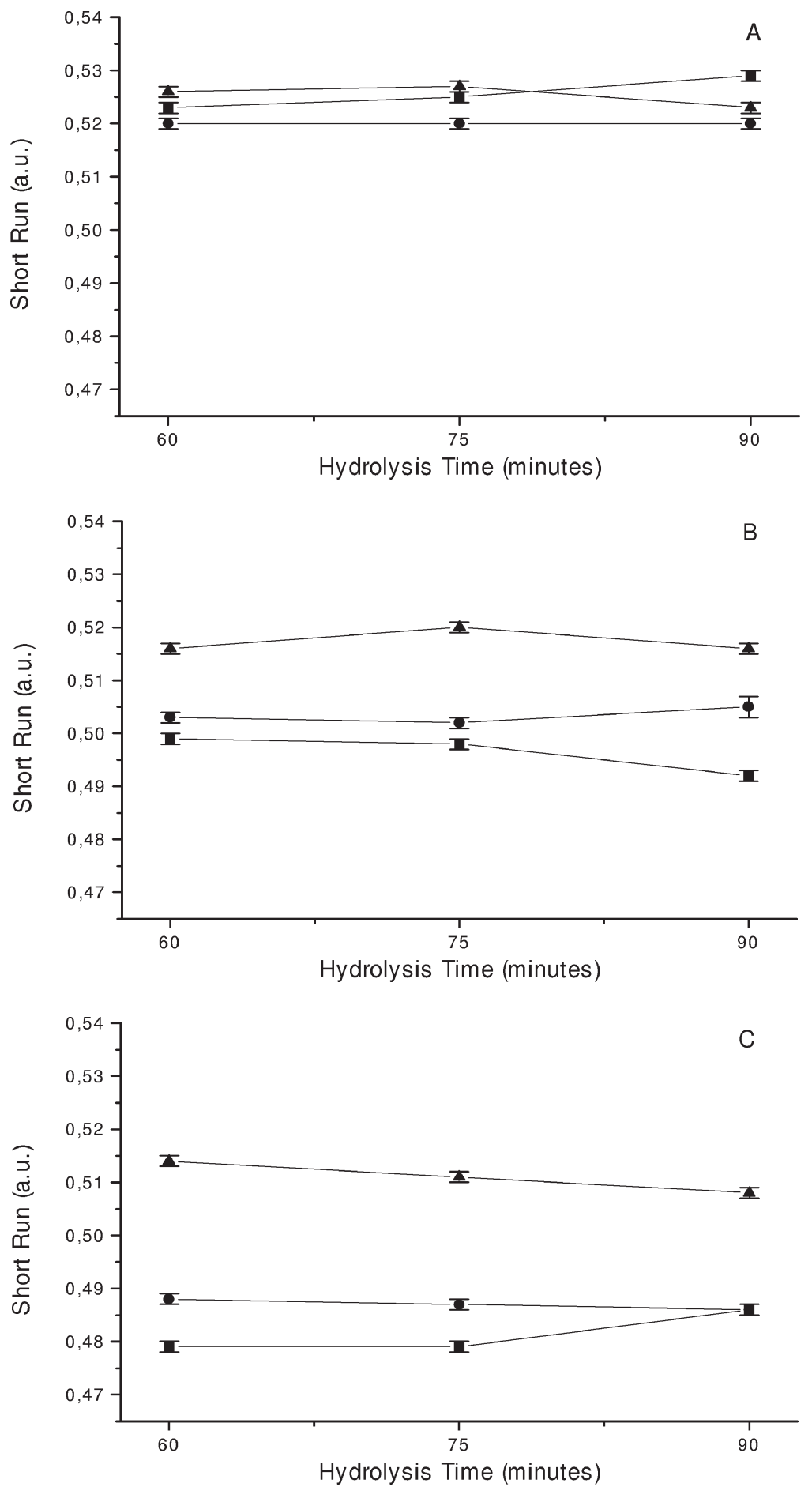

Fig. 6. Mean values $( \pm$ SEM) of short run in the plateau range of hydrolysis curve for lymphatic cells (A), epithelial cells from buccal mucosa (B) and uterine myometrial cells (C) fixed by different types of fixatives: - Delaunay, $\bullet$ - Saccomanno, $\Delta$ - air-drying with postfixation in modified Böhm-Sprenger fixative. 


\subsection{Run length feature: short run emphasis}

This feature gives maximal values to the nuclei with small chromatin clumps of different condensation. The values were constant between 60 and $75 \mathrm{~min}$ of hydrolysis for Delaunay fixed and for air-dried lymphatic cells (Fig. 6A), and for all three fixations of epithelial (Fig. 6B) and mesenchymal cells (Fig. 6C). For longer hydrolysis times the values were variable between fixations.

We found significant differences between different fixations at the same time of hydrolysis in lymphatic, epithelial and mesenchimal cells (Fig. 6), although there were some exceptions, such as no differences between the three fixations for lymphatic cells at $60 \mathrm{~min}$ and for mesenchymal cells at $90 \mathrm{~min}$. In all tissue types air-dried nuclei had highest values of this feature.

\section{Discussion}

It is widely accepted that any cytological samples can be used for image analysis. However, the samples should be postfixed in Böhm-Sprenger fixative to achieve satisfactory fixation before hydrolysis as a part of Feulgen staining. In routine clinical work, the cell samples for cytopathological examination are primarily either air-dried or fixed with different fixatives before any further staining, contrary to samples for histopathological examination where standard formalin fixation is used.

In our cytopathology department, the diagnostic cell samples are primarily fixed either by alcohol fixatives, i.e., Saccomanno (Table 1) or Delaunay fixative (Table 1), before Papanicolaou staining, or they are air-dried before Giemsa staining (which includes fixation with methanol). In our previous study we found significant differences in nuclear features, including nuclear texture features, between paired Papanicolaou and Giemsa stained fine needle aspirates of breast carcinomas, that were destained, and subsequently stained by the Feulgen method and analyzed by image cytometer [7]. The differences in nuclear feature values could be explained by the different effects of fixation before Papanicolaou or Giemsa staining, by different staining procedure, or even by the heterogeneity amongst cancer cells of the same tumour.

According to previous reports, both fixation and hydrolysis time affect significantly IOD values of nuclei that are stained using the Feulgen method and measured by image cytometry $[2,4,5,9,11-13$, $15,19,20]$. Only one report comments on the effect of different fixatives on nuclear texture features. The authors studied which of the fixatives used for Feulgen reaction gives the best results for image cytometric measurements. The criteria for a good fixative were a combination of nuclear stainability (IOD), perserved nuclear area and chromatin texture (reflected in nuclear texture features) [9]. In contrast, the aim of our study was to analyse and compare the effects of primary fixation, used in routine work, in cell samples that were subsequently postfixed in modified Böhm-Sprenger fixative (Table 1), as a part of the Feulgen procedure.

We found that both the primary fixation in combination with postfixation in modified Böhm-Sprenger fixative and the duration of hydrolysis in the IOD plateau range affect nuclear features. More interestingly, they affect nuclear texture features, which reflect the chromatin organization and structure as measured by image cytometers, despite the fact that the IOD is more or less constant over this range. In several instances, we found significant differences between nuclear texture feature values of nuclei of the same tissue, fixed by the same fixative, but with different hydrolysis times. Most frequently, we found such differences at $60 \mathrm{~min}$ (which is the time when IOD reaches plateau values on hydrolysis curve) and at $90 \mathrm{~min}$ of hydrolysis, i.e., at the outer limits of the plateau range. The values were generally constant between 60 and 75 min of hydrolysis in most of the cases, which indicates that the plateau 
range of the hydrolysis curve is shorter for the nuclear texture features than for the IOD values. According to the model described by Kjellstrand, the generation of aldehyde groups on the DNA molecule is a dynamic process of a formation of apurinic acid (which binds the stain) and loss of DNA fragments [11]. While the IOD is unchanged during the IOD plateau phase, there is a continuous generation and loss of stain-binding sites on DNA. Correspondingly, there is a continuous generation of binding sites in condensed (acid resistant) chromatin, leading to an increase of IOD and to pronounced staining of dense particles. At the same time the decondensed (acid sensitive) chromatin looses stain-binding sites, leading to a decreased IOD and to disappearance of small, lightly stained chromatin particles. Therefore the measurements of chromatin texture show constant values only in the beginning of the IOD plateau phase. Our observations suggest that the hydrolysis curve analysis should be done for all nuclear texture features and that one should select optimal hydrolysis time from the plateau range of nuclear texture features, depending on the primary fixation and tissue type.

The IOD of nuclei fixed in alcohol fixatives is higher than IOD of air-dried nuclei in all tissue types, which is in agreement with previous reports $[19,20,24]$. The possible explanation for higher values of alcohol fixed nuclei was offered in previous reports, which stated that alcohol precipitates the nuclear proteins and more binding sites are recovered on the DNA for the stain. Besides, TCA (tri-chlor acetic acid) in Delaunay fixative coagulates proteins and recovers additional binding sites, which gives even higher IOD values for the nuclei fixed in Delaunay fixative.

We found that the effect of different fixatives on nuclear area was different for nuclei of the three different tissue types. Most of the studies in the literature reported larger area of air-dried nuclei. In our study only the area of air-dried nuclei of lymphatic cells was higher than the area of alcohol fixed nuclei as reported before [19]. However, some authors reported that air-dried nuclei had smaller area, which is in agreement with our findings in mesenchymal cells [16]. Obviously, the air-drying has different effects on nuclear chromatin of different tissue types. The reason for the differences could be the different amount and type of cytoplasm, or possibly even the different chromatin structure and organisation, which both protect nuclear DNA and its structure during air-drying.

The effects of different fixations on discrete texture features, i.e., high density objects, were quite diverse among different tissue types and fixations. Since the effects of different fixations on other nuclear texture features were more consistent, we speculate that high density objects may not be most reliable feature for chromatin texture measurements. However, at least in lymphatic and mesenchymal cells, we were able to show that alcohol fixatives have similar effect on their nuclei.

As mentioned above, the effects of different primary fixations on the other groups of nuclear texture features are relatively constant for a given tissue type. The overall impression is that air-drying gives the largest values for entropy, fractal dimension and short run emphasis feature most of the time. However, in lymphatic cells, the values for the three features do not obey this "rule". We speculate that the thick chromatin network in these cells is more resistant to the effects of different fixatives.

In conclusion, we can say that nuclear texture features are sensitive to different primary fixations. Even though we used an identical, formalin type of postfixation before hydrolysis, as recommended in the Consensus Report of the ESACP [3], the effects of alcohol fixatives on nuclear texture features differ from air-drying effects. Further studies are needed to determine which of the primary fixations is better. The reproducibility of the results may not be the sole criterion, because different fixations have different effects on the nuclear features. It may be equally important to study which fixation affects features in a way that they correlate better with the diagnosis and prognosis of cancer patients. The studies performed on (archival or fresh) cytological material should report the type of primary fixation to ensure reliable comparison of the results between different laboratories, until the optimal primary fixation is defined. As 
shown in this study the chromatin texture is affected also by the hydrolysis times even during the plateau times of the hydrolysis curve for the IOD values. We suggest, therefore, that nuclear texture features need to narrow the selection of the optimal hydrolysis time, as if based only on the IOD results, one may choose a time inappropriate for other nuclear (texture) features.

\section{Acknowledgments}

This work was partially supported by the Slovenian Ministry of Science an Technology (grant J37956).

\section{References}

[1] B.F. Atkinson, Carbowax fixation of needle aspirates, Diagn. Cytopathol. 2 (1986), 231-232.

[2] M. Aubele, G. Burger and K. Rodenacker, Problems concerning the quality of DNA measurements on Feulgen stained imprints. A study of five fixation techniques, Analyt. Quant. Cytol. Histol. 16 (1994), 226-232.

[3] A. Böcking, F. Giroud and A. Reith, Consensus report of the ESACP task force on standardisation of diagnostic DNA cytometry, Anal. Cell. Pathol. 8 (1995), 67-74.

[4] R.L. Becker Jr. and U.V. Mikel, Interrelation of formalin fixation, chromatin compactness and DNA values as measured by flow and image cytometry, Analyt. Quant. Cytol. Histol. 12 (1990), 333-341.

[5] Y. De Launoit, R. Kiss and A. Danguy, Influence of smear preparation and fixatives on the DNA ploidy and morphonuclear features of the MXT mammary tumor and normal tissues in the mouse, Cytometry 11 (1990), 691-699.

[6] A. Doudkine, C. MacAulay, N. Poulin and B. Palcic, Nuclear texture measurements in image cytometry, Pathologica 87 (1995), 286-299.

[7] M. Fležar, B. Škrbinc and M. Us-Krašovec, The influence of previous fixation and staining on nuclear features of breast carcinoma cells, Anal. Cell. Pathol. 6 (1994), 232, Abstract 146.

[8] D.M. Garner, A. Harrison, C. MacAulay and B. Palcic, Cyto-Savant ${ }^{\mathrm{TM}}$ and its use in automated screening of cervical smears, in: Compendium on the Computerized Cytology and Histology Laboratory, Tutorials of cytology, Chicago, 1994, pp. 346-352.

[9] F. Giroud and M.P. Montmasson, Reevaluation of optimal Feulgen reaction for automated cytology. Influence of fixatives, Analyt. Quant. Cytol. Histol. 11 (1989), 87-95.

[10] B. Jaggi, S. Poon, B. Pontifex, J. Fengler and B. Palcic, A quantitative microscope for image cytometry, J. SPIE 1448 (1991), 89-97.

[11] P.T.T. Kjellstrand, Mechanisms of the Feulgen acid hydrolysis, J. Microscop. 119 (1980), 391-396.

[12] P.T.T. Kjellstrand, Temperature and acid concentration in the search for optimum Feulgen hydrolysis conditions, J. Histochem. Cytochem. 25 (1977), 129-134.

[13] V.M. Kotelnikov and L.L. Litinskaya, Comparative studies of Feulgen hydrolysis for DNA. I. Influence of different fixatives and polyethylene glycols, Histochem. 71 (1981), 145-153.

[14] C. MacAulay and B. Palcic, An edge relocation segmentation algorithm, Analyt. Quant. Cytol. Histol. 12 (1990), $165-171$.

[15] U.V. Mikel and R.L. Becker Jr., A comparative study of quantitative stains for DNA in image cytometry, Analyt. Quant. Cytol. Histol. 13 (1991), 253-260.

[16] N.M. Poulin, J.B. Matthews, K.A. Skov and B. Palcic, Effects of fixation method on cytometric measurement of DNA content and distribution in cells stained for fluorescence with propidium iodide, J. Histochem. Cytochem. 42 (1994), $1149-1156$.

[17] A. Reith and H. Danielsen, Assessment of DNA ploidy in tumor material. Preparation and measurement by image cytometry, in: Compendium on the Computerized Cytology and Histology Laboratory, G.L. Wied, P.H. Bartels, D. Rosenthal and U. Schenk, eds, Tutorials of cytology, Chicago, 1994, pp. 185-193.

[18] E. Schulte and C. Wittekind, The influence of wet-fixed Papanicolaou and the air-dried Giemsa techniques on nuclear parameters in breast cancer cytology. A cytomorphometric study, Diagn. Cytopathol. 3 (1987), 256-260.

[19] E. Schulte, Air-drying as a preparatory factor in cytology. Investigation of its influence on dye uptake and dye binding, Diagn. Cytopathol. 2 (1986), 160-167.

[20] E.K.W. Schulte and D.H. Wittekind, Standardization of Feulgen reaction. The influence of chromatin condensation on the kinetics of acid hydrolysis, Analyt. Cell. Pathol. 2 (1990), 149-157.

[21] E.K.W. Schulte, Standardization of biological dyes and stains: pitfalls and possibilities, Histochem. 95 (1991), 319-328. 
[22] P. van Duijin, A histochemical specific thionine- $\mathrm{SO}_{2}$ reagent and its use in a bicolor method for deoxyribonucleic acid and periodic acid-Schiff positive substances, J. Histochem. Cytochem. 4 (1956), 55-63.

[23] D. Wittekind, Standardization of dyes and stains for automated cell pattern recognition, Analyt. Quant. Cytol. Histol. 7 (1985), 6-29.

[24] G.C.H. Yang, The mathematical basis for the increased sensitivity in cancer detection in air-dried cytopreparations, Modern Pathol. 7 (1994), 681-684. 


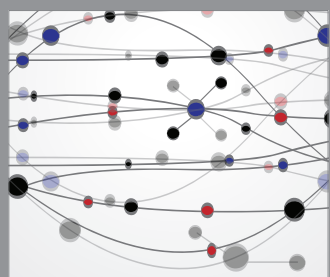

The Scientific World Journal
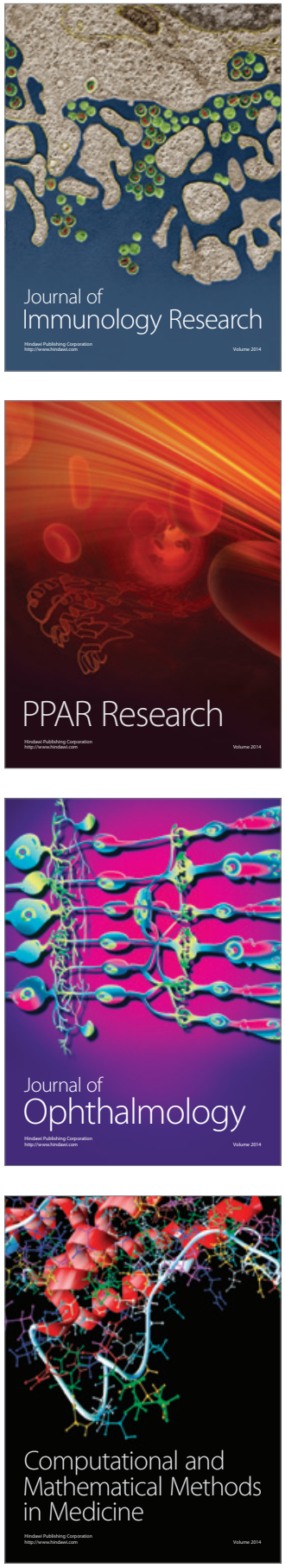

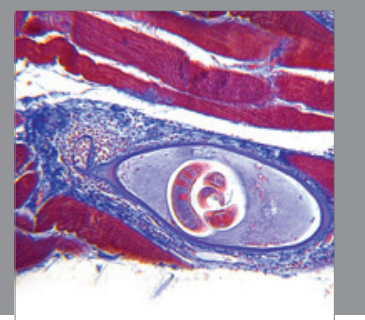

Gastroenterology

Research and Practice
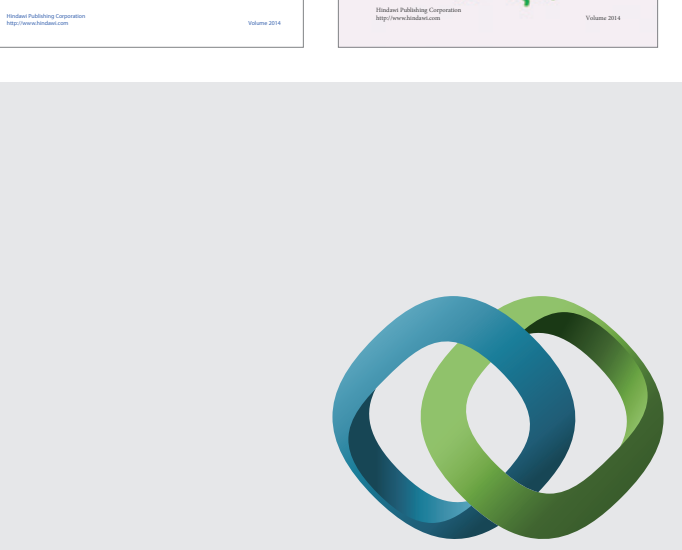

\section{Hindawi}

Submit your manuscripts at

http://www.hindawi.com
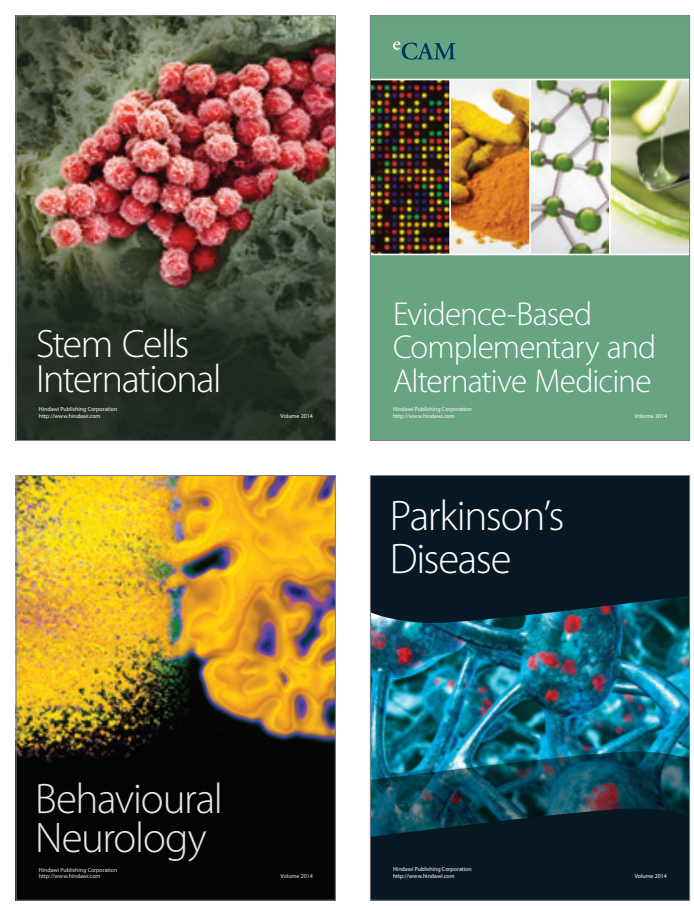

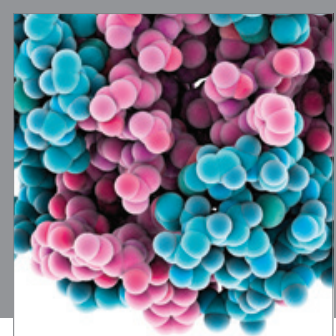

Journal of
Diabetes Research

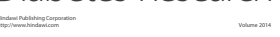

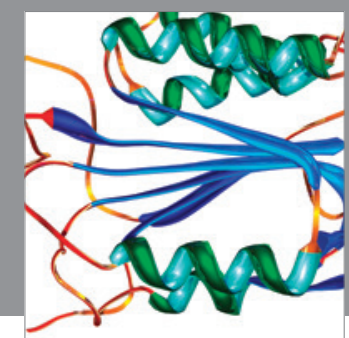

Disease Markers
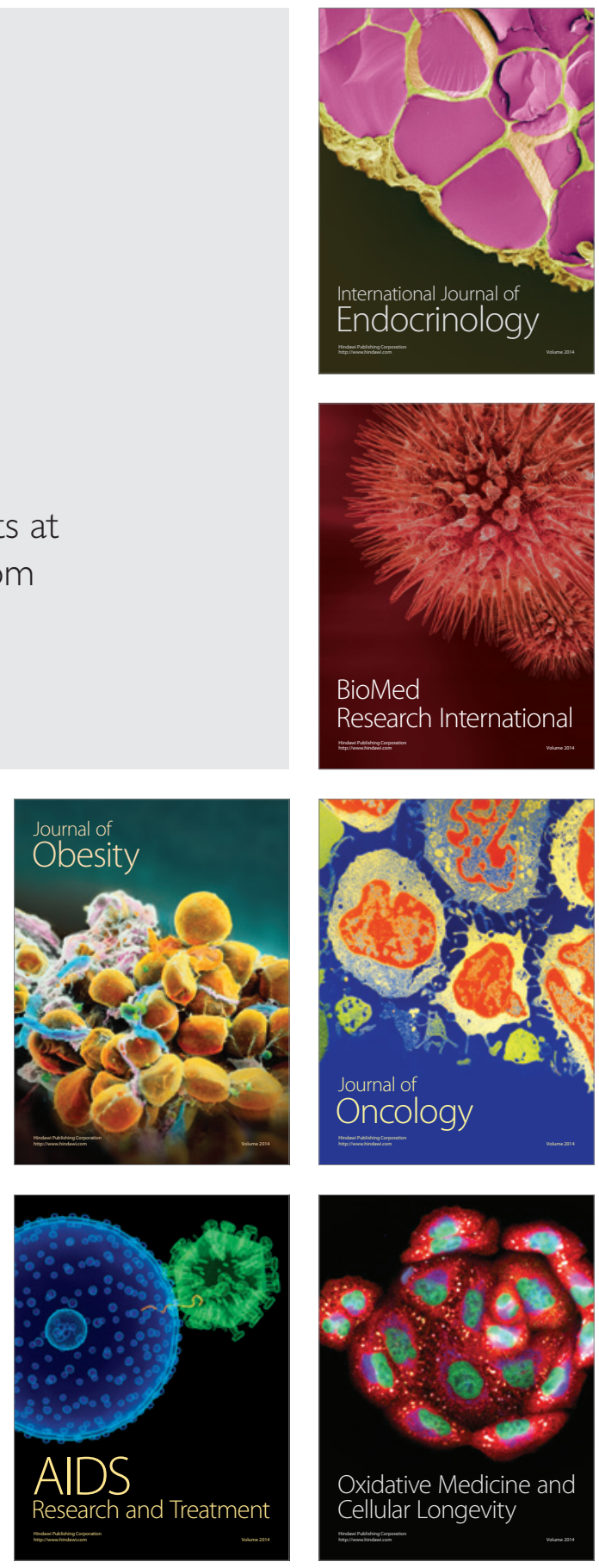